\title{
The Hybrid Model for Adaptive Educational Hypermedia.
}

\author{
Mohamed Ramzy Zakaria ${ }^{1}$, Adam Moore ${ }^{2}$, Helen Ashman ${ }^{1}$, Craig Stewart ${ }^{3}$ and Tim Brailsford ${ }^{1}$ \\ ${ }^{\mathbf{1} S c h o o l}$ of Information Technology and Computer Science, University of Nottingham, Nottingham, NG8 1BB, UK \\ mrz@cs.nott.ac.uk, \{helen.ashman, tim.brailsford\}@nottingham.ac.uk \\ ${ }^{2}$ School of Civil Engineering, University of Nottingham, Nottingham, NG7 2RD, UK \\ adam.moore@nottingham.ac.uk \\ ${ }^{3}$ School of Life and Environmental Sciences, University of Nottingham, Nottingham, NG7 2RD, UK \\ craig.stewart@nottingham.ac.uk
}

\begin{abstract}
Web-based distance learning is becoming increasingly prevalent as the Internet permeates every aspect of our culture, and many educational content management systems are now in use on the web. However, learners' experiences of these systems are almost invariably static, with information being delivered regardless of their background or knowledge. Due to variation between learners', it is suggested that these web-based distancelearning systems would benefit from the capability of adapting their content to meet individual needs. To effectively implement this adaptation of educational material, we require a user model that supplies the system with information about the learners using the system, such as their backgrounds, knowledge, interests and learning styles. This paper focuses on presenting a user model that combines the advantages of two techniques (overlay and stereotyping) in a way that provides the system with the ability to deliver information that is fully informed by the requirements of individual users.
\end{abstract}

Keywords: user modelling, adaptive hypermedia, educational systems, overlay, stereotyping

\section{Introduction}

The brave new era of the information age has ramifications for all disciplines, at the most fundamental of levels. From education, to commerce and music, the Internet impinges on every field where data and knowledge are currency. Arising out of this world-wide network of communications comes the globalisation of information - in which hypermedia tools are at the forefront enabling direct user access to information [1].

As the amount of information on the web continues its exponential increase, the number of users with different goals and interests also expands. It therefore becomes increasingly important that the information available be adapted to suit each user's individual knowledge and aspirations.

For example, in traditional web-based educational hypermedia systems, the contents are generally static, in so far as once written, their contents cannot be changed without external intervention. This provides a uniform learning experience to all learners, regardless of their needs and requirements. One example of such a system is WebCT [2]. This is a hypermedia educational system developed in 1995 at the University of British Columbia. It is an environment for authoring and delivering educational materials over the web. WebCT presents a static and inflexible pedagogic experience, without any kind of adaptation at the user level. Hence a web application, such as those delivered via WebCT, which are designed with a particular class of users in mind, may not suit those even marginally different from the original target audience [3].

In response to this clear need, adaptive hypermedia systems have been created such as AHA[4] and CHEOPS[5] . They build a model of the goals, interests, preference and knowledge of their users; so that they may present them with the information they need in a timely and appropriate manner [1].

This paper describes a hybrid user model, which is cooperative [6], (i.e. it collaborates with users in gathering information, as they are required to supply the system with some personal information, e.g. their occupation and preferences). This model also involves users in the user modelling process, as the contents of topics or courses are adapted according to their knowledge level about the topic they study. The hybrid model described below has the benefit that it should suit any adaptive educational hypermedia system, and we suggest that it is likely to provide a powerful addition to any technologybased learning programme. 


\section{The Hybrid Model}

\subsection{Architecture}

The hybrid model combines the use of two major techniques that are prevalent within the user modelling community. The first of these is the overlay model perhaps currently the most widely used technique of user modelling. This is used to measure the knowledge level of users in any given topic or domain. A user's knowledge according to this model is considered to be an overlay of the total knowledge representing that domain. This knowledge level is represented in the form of "ConceptValue" pairs, [7,8].

The second model is the stereotype; this technique assumes that knowledge is customised for specific groups, with each user being assigned to one and only one group at any given time. Thus, users who share the same background or knowledge should be assigned to the same group. Users can not change from one group (or class) to another until they trigger the specific conditions of the new group $[9,10]$. Aspects of each of these models are utilised by the hybrid model as follows:

- Overlay technique: the overlay measures the knowledge level of each learner within certain subject domains. This knowledge level might represent the score achieved in the system assessment at the end of each lesson, although any other parameters the system authors may choose may also be used. For example, the score achieved in self-assessment quizzes is a widely used and well-accepted metric of the comprehension of information.

- Level stereotype: level stereotypes mainly depend on the knowledge level of users. For example, they may simply be defined as Beginner, Intermediate and Advanced, but any classes may be used as appropriate to each system. According to the users knowledge level they will be assigned to a single class of the level stereotype within any given domain they of study. For example, a user studying biomechanics might be assigned simultaneously to the novice class in biology and to the advanced class in mathematics. Classes in the level stereotype are concerned with providing assistance that is appropriate, and adapting the contents of the lesson to suit the learner. Each class may define an article or set of articles, links to external documents, or to lessons in other courses. For example, if a user belongs to one of the advanced classes he may be provided with advanced articles or links to help the user to find more about the topic or domain he studies. Level stereotypes not only adapt the contents to suite a user's level, but they also facilitate learning by identifying domain weaknesses in the topic under study.

- Category stereotype: the hybrid model has been designed for systems that simultaneously run multiple courses for different levels of users. For example, the system may be running courses for first year undergraduate as well as postgraduate users. For this reason the users need to be categorised, as the knowledge level of undergraduate users in a certain stereotype level of a certain domain may not be the same as that of postgraduate users in the same stereotype level of the same domain. For example, consider two users, one of them a first year undergraduate and the second one a studying for a higher degree. Both of these students are classified in the intermediate level stereotype for the biology domain. Both of them are in the same level stereotype, but the intermediate level of postgraduates will be much more advanced than that of first year undergraduates. The categories stereotype helps the system to distinguish between different users in the same level stereotype, and to provide each of them the appropriate adaptation and help.

There is one other important aspect of this hybrid model - the information pool. This is categorised by the domain model, and consists of a pool of articles, links, and other items that encapsulate the resources of an adaptive system. The information pool is likely to differ in both form and content from one system to another.

The overlay technique, level stereotype and category stereotype combine to pick from the information pool the most convenient articles and links that suit each user's level, knowledge and background. Thus, according to a user's knowledge level and category, the most appropriate materials will be chosen from the information pool. Figure 1 shows the hybrid model's components, comprising of two stereotypes and one overlay, to provide the maximum flexibility and to have the capability to serve a wide range of users. 


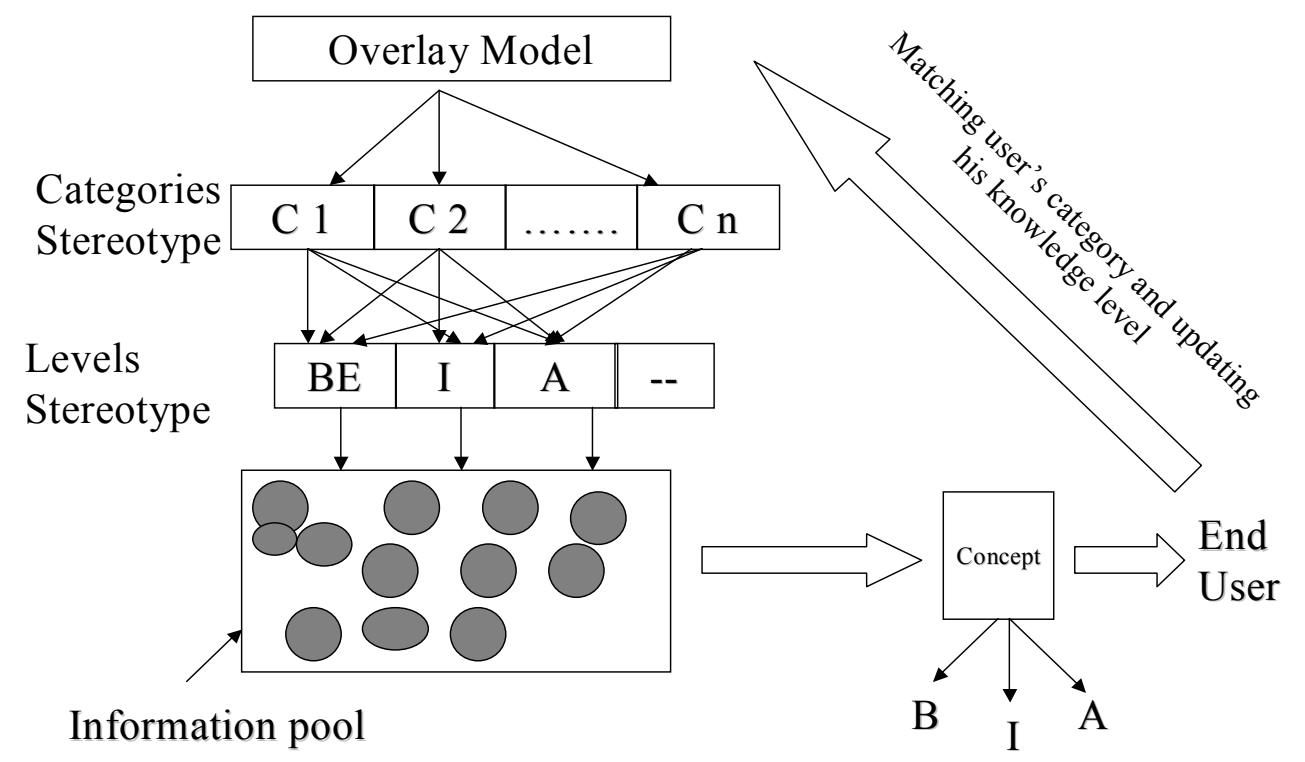

Fig. 1. The components of the hybrid model. An overlay model combines with a category and level stereotype to retrieve appropriate content from the information pool to convey concepts to the user. The user interacts with the system to inform and update the user model. BE - Beginner, B - Basic, I - Intermediate, A - Advanced.

\subsection{Mechanism}

When a user logs on to the system for the first time, he/she will be given an initial knowledge level value according to an estimate of prior knowledge about the subject under study. That recommends the user to a certain level stereotype, and the category is determined according to any parameters that the system authors may choose such as user's occupation. Each time a user passes from one lesson to another the knowledge level for that user is updated according to the score in the system assessment (as well as other parameters the system's authors may choose). According to the user's new knowledge level, the class assigned according to the level stereotype might be changed or might be the same (i.e. if the user still has the same knowledge level). The adaptation of the contents and the supporting articles are available according to the class of the user in the level stereotype as well as the category. The steps involved in adaptation are illustrated in Figure 2.

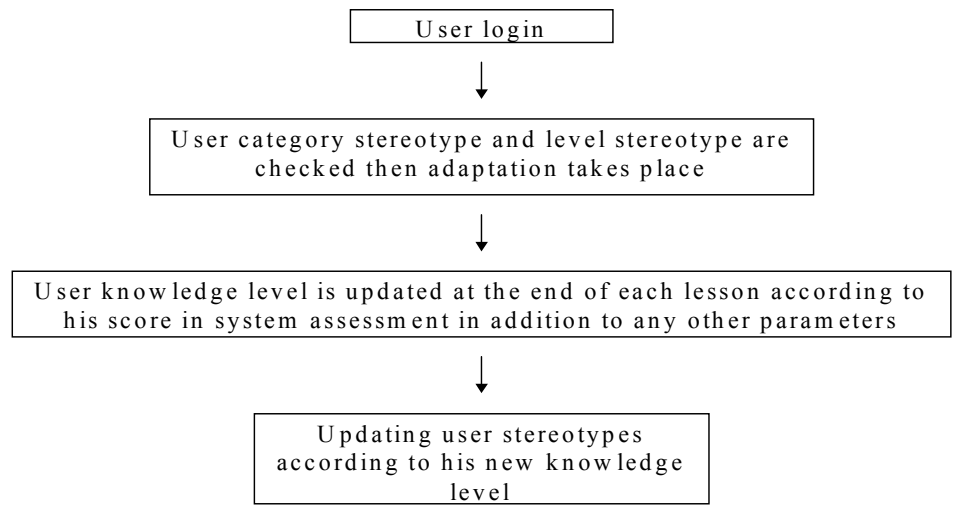

Fig. 2. The hybrid model mechanism to adapt materials to users. Once the user logs in, the system either adapts its material according to the existing user model, or creates a new one. At the end of each lesson, the user model is updated. 


\section{Implementation of the hybrid model in a hypermedia learning environment}

The hybrid model is currently being integrated into an adaptive educational hypermedia system called WHURLE (Web-based Hierarchal Universal Reactive Learning Environment), $[11,12,13]$ an XMLbased integrated learning environment. In WHURLE the content consists of atomic chunks, each of which consists of the smallest conceptually self-contained unit of information that the author can envisage. The domain of WHURLE content is contained within the melange, which consists of all the available chunks in any single installation. In WHURLE conditional transclusion [14] is used to construct virtual documents, which contain one or more of these chunks. The hybrid model is integrated into WHURLE as the filter for the lesson plan. This filter generates an adapted virtual document dependant upon both the adaptation rules within the lesson plan and user profile. WHURLE lesson plans represent the information pool of the hybrid model $[11,12,13]$.

\section{Conclusion}

The hybrid model is a user model that gathers together the most commonly used techniques of usermodelling for adaptive hypertext. This utilises the advantages of each of these techniques in a way to provide a full understanding for the user's needs and requirements on several different levels. Using this technique we have implemented adaptation within WHURLE to allow students to see pages of information containing one or more chunks in a manner relevant to their skills, knowledge and learning styles, following rules set by the author of the lesson that they are viewing. Using this model we hope that the WHURLE system will provide a strong pedagogic framework for a variety of web-based learning scenarios.

\section{Acknowledgements}

We wish to thank Peter Murray-Rust, Peter Davies, and Ban Seng Choo for many useful discussions, and colleagues in the WTG, VSB and IBiS for their support and encouragement. Craig Stewart is a research associate funded by the Hong Kong University Grants Committee.

\section{References}

[1] Brusilovsky, P. (1996). "Methods and techniques of adaptive hypermedia". User modeling and user-adapted interaction, 6(2-3), pp. 87-129.

[2] Beshears, Fred. "WebCT overview". http://socrates.berkeley.edu:7521/articles/webct/WebCT-Presentation

[3] Eklund,J; Brusilovsky,P; Schwarz,E.(1997). "Adaptive Textbooks on the World WideWeb”, Proceedings of AUSWEB97, the third Australian conference on the world wide web, Queensland, Australia, July 5-9, 1997, Southern Cross University press, pp. 186-192 .http://ausweb.scu.edu.au/proceedings/eklund/paper.html. [4]De Bra,P;Calvi,L.(1998). "AHA Adaptive Hypermedia Architecture".NRHM journal, V.4, pp 115 - 139 [5] Ferrandino,S; Negro,A;Scarano,V.(1997). "CHEOPS :Adaptive Hypermedia on the World Wide Web”. Proceedings of the European Workshop on Interactive Distributed Multimedia Systems and Telecommunicazion Services (IDMS '97).

[6] Kay, J. 1995, “The UM toolkit for cooperative user models”. User Models and User Adapted Interaction 4(3), 149-196.

[7] Valley,K. (1997). "Learning Styles and Courseware Design". Association of Learning Technology Journal, 5(2), p42-51

[8] Carr,B; Goldstein, I. (1977). "Overlays, a theory of modelling for computer aided instruction". A technical report. AI memo 406, MIT, Cambridge, MA.

[9] Rich, E. (1983). "Users are individuals: individualizing user models", Journal of man-machine studies vol.18, $199-214$.

[10] Benaki,E; Karkalestsis,V; Spyropoulos,C. (1997). "User modelling in WWW: the UMIE prototype". Proceedings of sixth international conference on user modelling, Chia Laguna, Sardinia, 2-5 June1997 http://www.contrib.andrew.cmu.edu/ plb/UM97 workshop/Benaki/Benaki.html

[11] Brailsford, TJ ; Moore, A ; Stewart, CD ; Zakaria, MR ; Choo, BS ; Davies, PMC.(2001). "Towards a framework for effective web-based distributed learning". WWW10 proceedings, HongKong.

[12] Moore,A; Brialsford,T.J, Stewart, C.D. (2001). "Personally tailored teaching in WHURLE using conditional transclusion". The twelfth ACM conference on hypertext and hypermedia. August 14-13, 2001, Denmark.

[13] Brailsford,T; Stewart,C; Zakaria,M; Moore,A.(2002).”Autonavigation, Links and Narrative in an Adaptive Web-Based Integrated Learning Environment". Proceedings of www2002, Hawaii, USA.

[14] Nelson, T.H. (1995). "The Heart of Connection: hypermedia unified by transclusion". Communications of the ACM. 